\title{
Numerical simulation of the blocking process of gelled particles in porous media with remaining polymers
}

\author{
Feng Qihong ${ }^{1 *}$, Zhang Ge ${ }^{1}$, Yin Xiaomei ${ }^{1}$ and Luan Zhiyong ${ }^{2}$ \\ ${ }^{1}$ School of Petroleum Engineering, China University of Petroleum, Dongying, Shandong 257061, China \\ ${ }^{2}$ Oil Production Research Institute, Shengli Oilfield Company, Sinopec, Dongying, Shandong 257000, China
}

\begin{abstract}
Gelled particles can be transferred deeply inside oil reservoirs to block water channels due to their physicochemical characteristics, including swelling, deformation, and synergetic effect (reacting with polymers), and then the injection profiles are significantly modified. At present, research on gelled particles is mainly focused on laboratory studies of drive mechanisms, and rarely on mathematical models describing the blocking process of gelled particles. In this paper, the blocking process of gelled particles is divided into two sub-processes: deposition and desorption due to particle deformation. A mathematical model based on filtration theory is proposed considering the effect of characteristics of gelled particles on the blocking process. Blocking laws were simulated and researched using the mathematical model. Results of the simulation of the blocking of gelled particles are quite consistent with the experimental results, which confirms the reliability of the mathematical model developed.
\end{abstract}

Key words: Gelled particles, profile control, blocking process, numerical simulation, swelling, synergetic effect, deformation

\section{Introduction}

Polymer flooding is commonly used to improve water sweep efficiency in oil reservoirs. However, oil recovery is relatively low due to serious water channeling when polymer flooding is used in oil reservoirs with high-permeability channels. In order to plug these high-permeability channels, blocking agents are injected to modify the injection profiles in high-permeability reservoirs and divert following fluids (normally, water) to low-permeability zones. An effective blocking agent (Liu and Bai, 2006), gelled particles, gradually attract more attention in the field of chemical drive. Research on gelled particles is mainly focused on laboratory studies of drive mechanisms (Chauveteau et al, 2000; 2001; Lu and Cao, 2004), and rarely focused on mathematical modeling of the blocking process. In this paper, a mathematical model of the blocking process of gelled particles is proposed. This is of significance for scheme selection and parameter optimization.

\section{Mechanisms of improvement of injection profiles by gelled particles}

Gelled particles are obtained by crushing dry gel, which is made by polymerization and crosslinking of polymer monomer, crosslinker, and other additives under specific conditions ( $\mathrm{Li}$ et al, 1999; Bai et al, 2004; Hu et al, 2006). As gelled particle suspensions flow through porous media,

** Corresponding author. email: fengqihong@126.com

Received December 30, 2008 physicochemical behavior of the gelled particles is very complex, including swelling, deformation, and synergetic effect (reacting with polymers remaining in porous media). The gelled particle has ability to deform compared with a rigid particle, so it is called an "amoeba".

When gelled particles are injected into a reservoir, three major mechanisms will take place: (1) Gelled particles swell after contact with water, resulting in increasing equivalent volume. (2) Gelled particles may be deposited in narrow throats to plug the macropores when the pressure gradient is lower than breakthrough pressure gradient of particles. While the pressure gradient is higher than breakthrough pressure gradient, gelled particles will deform to pass through narrow throats and flow deeply inside the reservoir, thus modifying the injection profile (Coste et al, 2000; Yue et al, 2006). (3) Moreover, gelled particles react with polymers remaining in the reservoir to form floc units, therefore, the equivalent volume of gelled particles further increases.

\section{Numerical simulation of the blocking process of gelled particles in porous media}

Gelled particles remain in the reservoir and then plug macropores. This mechanism is very similar to the percolation of sewage (Katz, 1982). Some researchers have applied this theory to investigating reservoir damage (Liu and Hou, $1995)$ and percolation characteristics of suspending liquid in reservoirs (Zhang and Liu, 2007). However, the abovementioned studies had obvious deficiencies for building the 
continuity equation. So the continuity equation was rebuilt in this paper and applied to developing a new mathematical model of gelled particles used to control injection profiles, on the basis of plane radial flow theory.

\subsection{Kinematic equation of gelled particles}

\subsubsection{Kinematic equation for the deposition process}

Assuming that the blocking agent used to modify injection profiles is a dispersion system of gelled particles, particle deposition in porous media can be described with a kinematic equation of filtration, which can be written in the following general form (Liu, 1994):

$$
\frac{\partial C}{\partial r}=-\lambda C
$$

where $C$ is the mass concentration of gelled particles in the suspension, $\% ; r$ is the distance from the injection site, $\mathrm{m} ; \lambda$ is the filter coefficient, $1 / \mathrm{m}$, which is defined as a measurement of filtration efficiency and can be written in the following general form:

$$
\lambda=\lambda_{0}\left(1+\frac{\alpha \delta}{m}\right)^{y}\left(1-\frac{\delta}{m}\right)^{z}\left(1-\frac{\delta}{\delta_{\max }}\right)^{x}
$$

The general formula used in this study was proposed by Maroudas and Eisenklam (1965):

$$
\lambda=\lambda_{0}\left(1-\frac{\delta}{\delta_{\max }}\right)
$$

where $\lambda_{0}$ is the initial filter coefficient, $1 / \mathrm{m} ; x, y$ and $z$ are experimental indices, dimensionless; $\alpha$ is an experimental coefficient, dimensionless; $m$ is a geometry constant related to filler grain sizes of a clean medium, dimensionless; $\delta$ is the specific deposit (or reduced porosity), which is defined as the volume of deposited particles per unit apparent volume of rock; $\delta_{\max }$ is the saturation value of specific deposit, dimensionless.

\subsubsection{Kinematic equation for the deformation and desorption process}

Gelled particles will desorb from the surface in porous media due to their deformation at a pressure gradient higher than the breakthrough pressure gradient. No special kinematic equation has been available to describe this process. In this study, the kinematic equation considering deformation and desorption is derived from following experimental results. Assuming that the breakthrough pressure gradient of gelled particles is $p_{\text {Gmax }}$, the quantity of gelled particles released from the surface in porous media is proportional to the pressure gradient, Darcy velocity, time and specific deposit parameter according to experimental results. Therefore, the difference equation describing the variation of specific deposit caused by deformation and desorption of gelled particles at any point in the reservoirs can be expressed as follows:

$$
\delta_{i}^{n+1}=\delta_{i}^{n}-\Psi \delta_{i}^{n} v \Delta t\left(p_{\mathrm{G}}-p_{\mathrm{Gmax}}\right)
$$

where $\Psi$ is the desorption coefficient and can be obtained by experiments, dimensionless; $p_{\mathrm{G}}$ is the driving pressure gradient, $\mathrm{MPa} / \mathrm{m} ; v$ is the filtration rate, $\mathrm{m} / \mathrm{min} ; t$ is the filtration time, $\min$.
The above equation can be expressed in the differential form:

$$
\frac{\partial \delta}{\partial t}=-\Psi \delta v \frac{\partial p}{\partial r}
$$

Eq. (2) is the kinematic equation which describes the desorption process of gelled particles due to deformation.

\subsection{Continuity equation of gelled particles}

In order to construct the continuity equation, taking an annular element and assuming that the filtration flow area is $A$, the element volume $V_{\mathrm{b}}$, the concentration of gelled particles in the suspending liquid $C$, the filtration rate $v$, the simulation time $\Delta t$, the porosity of a clean medium $\phi$, the specific deposit $\delta$, and the volumetric concentration of suspending liquid in injection/production element in unit time is $q$.

According to conservation of mass:

[Inflow mass of particles in the element]-[outflow mass of particles in the element] + [mass of injected/produced particles in the element $]=[$ incremental mass in the element $]+[$ mass variation of particles in the suspending liquid in the element]. Assuming that the density of particles is unchanged, the difference form of the continuity equation is obtained by removing the density term:

$$
\left.v A C\right|_{r} \Delta t-\left.v A C\right|_{r+\Delta r} \Delta t+q=V_{b} \Delta \delta+V_{b} \Delta(\phi C)
$$

with

$$
\begin{aligned}
& A=2 \pi r h \\
& V_{\mathrm{b}}=\pi(r+\Delta r)^{2} h-\pi r^{2} h=2 \pi r h \Delta r+\pi h \Delta r^{2} \approx 2 \pi r h \Delta r
\end{aligned}
$$

By rearranging the above equation, we get the continuity equation in the differential form:

$$
-\frac{1}{r} \frac{\partial(r v C)}{\partial r}+q=\frac{\partial \delta}{\partial t}+\frac{\partial(\phi C)}{\partial t}
$$

\subsection{Mathematical model and numerical solution}

A mathematical model for describing gelled particles to modify injection profiles is constructed using Eqs. (1), (2), (3), and initial and boundary conditions.

$$
\left\{\begin{array}{l}
\frac{\partial C}{\partial r}=-\lambda C \quad p_{\mathrm{G}} \leq p_{\mathrm{Gmax}} \\
\frac{\partial \delta}{\partial t}=-\Psi \delta v\left(\frac{\partial p}{\partial r}-p_{\mathrm{Gmax}}\right) \quad p_{\mathrm{G}}>p_{\mathrm{Gmax}} \\
-\frac{1}{r} \frac{\partial(r v C)}{\partial r}+q \\
=\frac{\partial \delta}{\partial t}+\frac{\partial(\phi C)}{\partial t} \quad \text { (injection flow rate } q \text { is positive) } \\
\text { Initial boundary condition: } C(0, t)=C_{0} \\
\text { Initial condition: } \delta(r, 0)=0 \quad C(r, 0)=0 \\
\lim _{t \rightarrow \infty} \delta(r, t)=\delta_{\max } \quad \lambda=\lambda_{0}\left(1-\delta / \delta_{\max }\right)
\end{array}\right.
$$


The iteration formula of solution can be obtained by discrete difference approximations of the above mathematical model. Details about this process are not given here, but are available from the authors on request.

\section{Dynamic model of poroperm parameters}

Gelled particles injected into reservoirs flow preferentially into high-permeability channels and particle deposition or retention reduces the porosity and permeability of reservoirs, thus modifying injection profiles. The equivalent volume of retained particles increases because of the swelling of gelled particles and the synergistic reaction between polymers. Therefore, the swelling coefficient $a$ and synergic effect coefficient $b$ are introduced to modify the specific deposit $\delta$. The modified specific deposit is $a b \delta$, where $a$ and $b$ are obtained by experiments.

Gelled particle retention causes changes in reservoir porosity and permeability. Instantaneous porosity can be expressed as follows:

$$
\phi(r, t)=\phi_{0}-a b \delta(r, t)
$$

where $\phi_{0}$ is the initial porosity.

Instantaneous permeability can be expressed as follows (Liu and Civan, 1993; Liu and Hou, 1995):

$$
\begin{aligned}
& k(r, t)=k_{0}\left[(1-f) D+f \frac{\phi(r, t)}{\phi_{0}}\right]^{3} \\
& f=1-\beta a b \delta(r, t)
\end{aligned}
$$

where $k_{0}$ is the initial permeability of the media, $10^{-3} \mu \mathrm{m}^{2}$; $f$ is the flow efficiency factor, defined as effective flow fraction, dimensionless; $D$ is the allowed constant of filtration after plugging, dimensionless; $\beta$ is a filtration constant, dimensionless.

\section{Applications}

A plane radial flow model of a single well is built to investigate the blocking of gelled particles. The inner boundary is specified by the injection rate and the outer boundary is specified by the pressure of one standard atmosphere. The static parameters of the model are shown in Table 1 . These data are modified from oil field data.

Table 1 Static parameters of plane radial flow model

\begin{tabular}{ccccc}
\hline $\begin{array}{c}\text { Wellbore radius } \\
\mathrm{m}\end{array}$ & $\begin{array}{c}\text { Model radius } \\
\mathrm{m}\end{array}$ & $\begin{array}{c}\text { Model thickness } \\
\mathrm{m}\end{array}$ & Porosity & $\begin{array}{c}\text { Permeability } \\
10^{-3} \mu \mathrm{m}^{2}\end{array}$ \\
\hline 0.1 & 100 & 1 & 0.25 & 400 \\
\hline
\end{tabular}

Coefficients used in simulation are shown in Table 2. $D$ and $\beta$ are the data quoted from the literature (Zhang and Liu, 2007). $a$ and $b$ are determined by swelling experiments of gelled particles. $\lambda_{0}$ is obtained by measurement of the filter coefficient (Zuo, 1998). The calculation process of $\Psi$ is so complicated that some experiments are firstly conducted to measure specific retention volume, driving pressure gradient, breakthrough pressure gradient, and flow velocity of gelled particles at different times. It can be calculated using Eq. (2).
Table 2 Coefficients in calculation

\begin{tabular}{ccccccc}
\hline$D$ & $\beta$ & $a$ & $b$ & $\lambda_{0}$ & $\Psi$ & $\delta_{\max }$ \\
\hline 0.15 & 3.5 & 11.0 & 11.0 & 0.01 & 0.01 & 0.18 \\
\hline
\end{tabular}

\subsection{Impact of injection time on blocking process of gelled particles}

The concentration of gelled particles in suspending liquid is specified as $4 \%$ and the injection rate is specified as $20 \mathrm{~m}^{3} /$ d. Assuming that the breakthrough pressure gradient of gelled particles is $3 \mathrm{MPa} / \mathrm{m}$, the distribution of pressure gradient, permeability, and specific deposit are simulated at different injection times and the results are shown in Figs. 1-3.

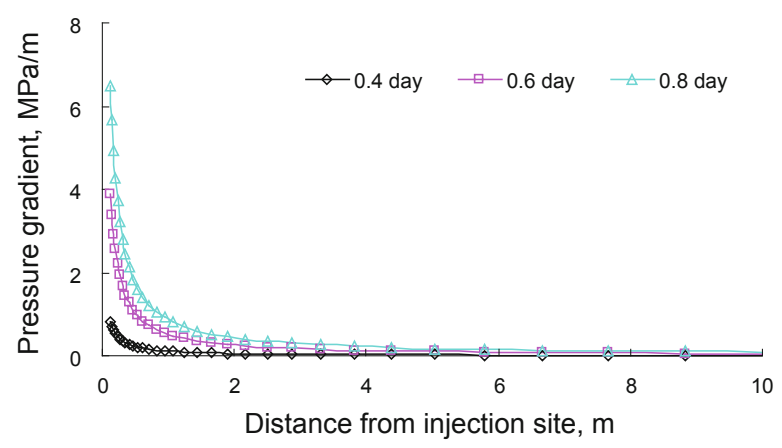

Fig. 1 Distribution of pressure gradients at different injection times

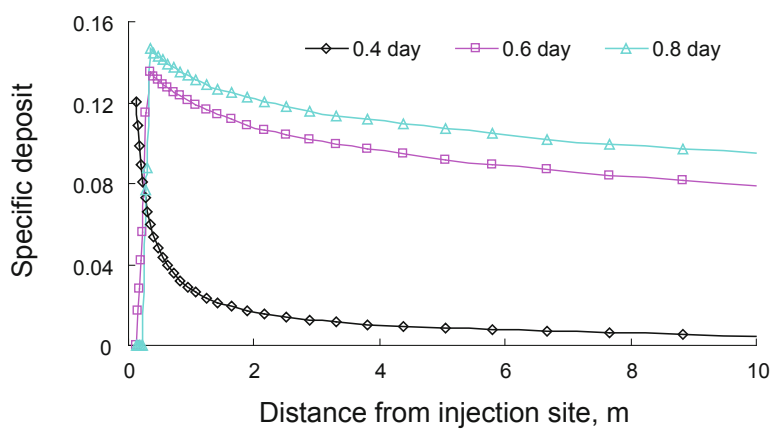

Fig. 2 Particle specific deposit at different injection times

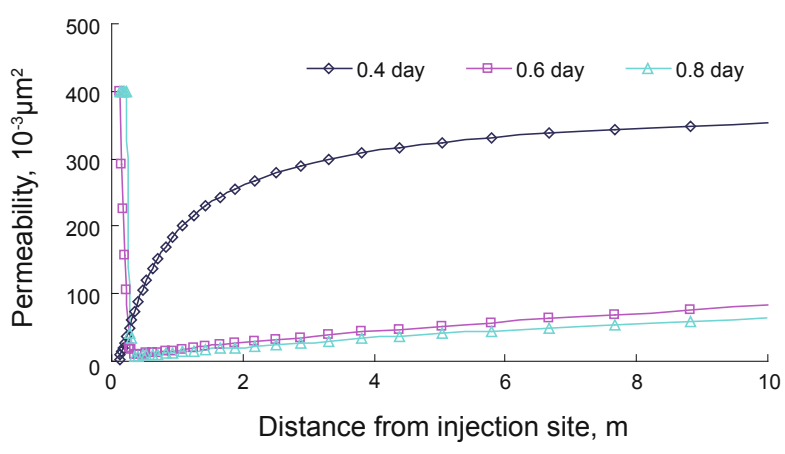

Fig. 3 Permeability distribution at different injection times 
Figs. 1-3 indicate that injected gelled particles accumulate in the reservoir when pressure gradient is not higher than the breakthrough pressure gradient. The nearer to the injection well, the larger the number of particles that accumulate, and the greater the extent of permeability reduction. When the pressure gradient is higher than the breakthrough pressure gradient, gelled particles may deform and desorb from the surface in porous media and flow deeply inside the reservoir. Therefore, the pressure gradient is higher in the immediate vicinity of injection wells than other zones due to the pressure cone around the wells. After injecting over a period of time, particle deposition will decrease in the regions close to wellbore, which causes an increase in permeability. At this time, particle deposition can be compared to a wave crest moving towards deep parts of the reservoir. As a result, a decrease in particle deposition in the immediate vicinity of wellbore does not lead to significant permeability reduction, this is also conducive to further injection of gelled particles.

\subsection{Impact of injection concentration on blocking process of gelled particles}

The injection rate is specified as $20 \mathrm{~m}^{3} / \mathrm{d}$ and injection time is specified as 0.6 day. Particle deposition is studied when the concentrations of gelled particles in suspending liquid are $4 \%, 5 \%$, and $6 \%$, respectively. The simulation results as shown in Fig. 4 indicate that the higher the particle concentration, the better the plugging efficiency at the same injection rate and time interval. The wave crest propagates deep inside the reservoir and there is a maximum value of particle deposition. When the concentration of gelled particles increases to $6 \%$, a plateau appears on the curve. The reason of the above-mentioned results is that an increase in the concentration of gelled particles in the suspending liquid will make more gelled particles transfer into the reservoirs, thus achieving a high plugging efficiency. This leads to a high injection pressure and a consequential increase in the driving pressure gradient near the injection well, and then more particles migrate deeply inside the reservoir. Maximum particle deposition exists when wave crest travels deep inside the reservoir. When particle deposition is over this value, gelled particles are no longer retained.

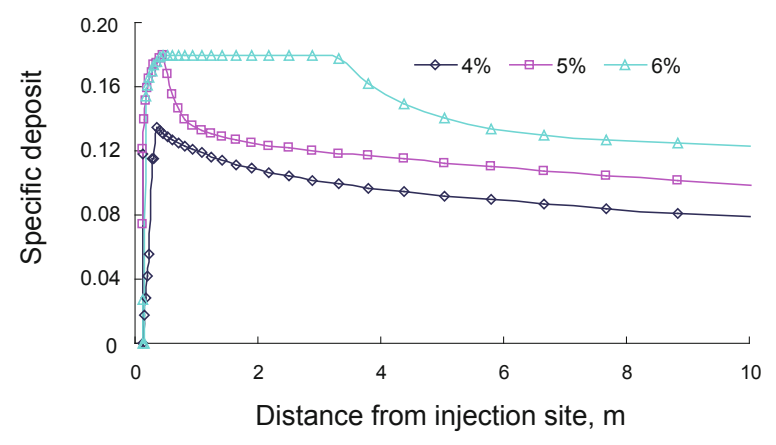

Fig. 4 Particle specific deposit at different concentrations of particles in suspending liquid

\subsection{Impact of injection rate on the blocking process of gelled particles}

The concentration of gelled particles in suspending fluid is
$4 \%$ and injection time is 0.6 day, particle retention is studied when the injection rates are 20,22 , and $24 \mathrm{~m}^{3} / \mathrm{d}$, respectively. Simulation results (as shown in Fig. 5) illustrate that the gelled particles have more effective plugging ability at higher injection rates with the other conditions the same. The reason is the same as the impact of concentration on the blocking process of gelled particles. With increasing injection rates of suspending liquid of gelled particles, large numbers of gelled particles flow into the reservoir, and deposit in the channels, leading to an increase in injection pressure. This indicates that the driving pressure gradient in the immediate vicinity of wellbore is higher than the breakthrough pressure gradient, then more gelled particles migrate deeply inside the reservoir.

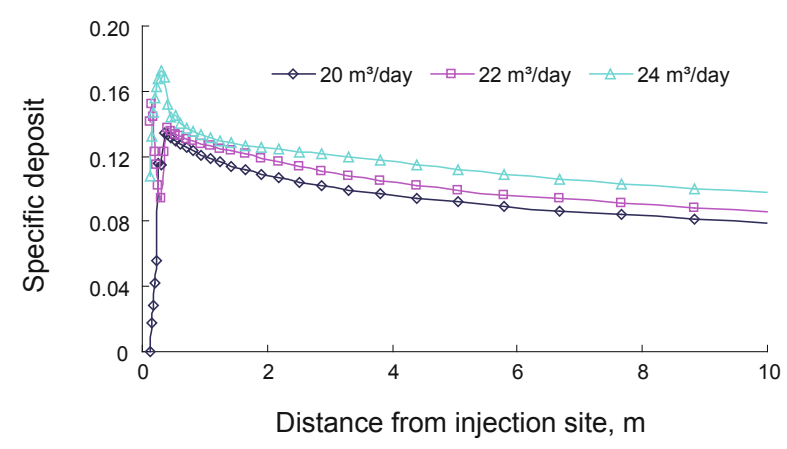

Fig. 5 Particle specific deposit at different injection rates

\section{Conclusions}

1) Profile control by gelled particles is divided into two sub-processes, particle deposition and particle desorption due to its deformation. Kinematic equations for both subprocesses are developed and a mathematical model for describing blocking process by gelled particles is constructed on the basis of filtration theory.

2) The mathematical model developed in this study for plane radial flow model of a single well has been applied to the blocking process of gelled particles.

3) Mechanisms of gelled particles to modify injection profile after polymer flooding are complex. In this paper, research focuses on some main mechanisms of gelled particles. The mathematical model is developed considering the blocking characteristics of gelled particles. The simulation results are in accordance with experimental results.

4) Profile control by gelled particles can reduce the permeability of reservoirs and then influence filtration of multiphase fluid in reservoirs. Based on the developed model of profile control by gelled particles and the filtration model in reservoirs, further research on filtration of multiphase fluid during profile control can be made in the future, as it is of great significance to the development of numerical simulation of profile control by gelled particles.

\section{Acknowledgements}

This work was supported by the National High Technology Research and Development Program of China (863 Program) (2007AA06Z214) and "Taishan Scholars" Construction Project (No. ts20070704). 


\section{References}

Bai B J, Li L X, Liu Y Z, et al. Preformed particle gel for conformance control: factors affecting its properties and applications. SPE/DOE Symposium on Improved Oil Recovery held in Tulsa, Oklahoma, 1721 April, 2004 (SPE paper 89389)

Chauveteau G, Omari A, Tabary R, et al. Controlling gelation time and microgel size for water shutoff. SPE/DOE Improved Oil Recovery, held in Tulsa, Oklahoma, 3-5 April, 2000 (SPE paper 59317)

Chauveteau G, Omari A, Tabary R, et al. New size-controlled microgels for oil production. SPE International Symposium on Oilfield Chemistry, held in Houston, Texas, 13-16 February, 2001 (SPE paper 64988)

Coste J P, Liu Y, Bai B J, et al. In-depth fluid diversion by pre-gelled particle: laboratory study and pilot testing. SPE/DOE Improved Oil Recovery Symposium, held in Tulsa, Oklahoma, 3-5 April, 2000 (SPE paper 59362)

Hu S Y, Zhang L H and Yu H J. Development and prospect of the profile control/water shutoff technology in reservoir high-capacity channels. Drilling \& Production Technology. 2006. 29(6): 117-119 (in Chinese)

Katz T M. Physicochemical Processing of Water (1st edition). Translated by Li W Y. Beijing: Tsinghua University Press. 215-223 (in Chinese)

Li Y X, Liu Y Z, Bai B J, et al. Research on expansion particle chemical for water shut-off and profile control. Oil Drilling \& Production Technology. 1999. 21(3): 65-68 (in Chinese)

Liu S. Mathematical model and analysis of filtration systems. Acta
Mathematica Scientia. 1994. 14(4): 361-365 (in Chinese)

Liu X H and Civan F. Characterization and prediction of formation damage in two-phase flow systems. Production Operations Symposium held in Oklahoma City, OK, U.S.A., 21-23 March, 1993 (SPE paper 25429)

Liu X P and Hou L P. A mathematical model for simulating formation damage due to solid particle invasion during water injection. Journal of Jianghan Petroleum Institute. 1995. 17(2): 69-73 (in Chinese)

Liu Y Z and Bai B J. Application and development of chemical-based conformance control treatments in China oilfields. SPE/DOE Symposium on Improved Oil Recovery held in Tulsa, Oklahoma, U.S.A., 22-26 April, 2006 (SPE paper 99641)

Lu F S and Cao S Y. Modeling experiments on controlling profile by gelled particles. Oil \& Gas Recovery Technology. 2004. 11(6): 61-63 (in Chinese)

Maroudas A and Eisenklam P. Clarification of suspension: a study of particle deposition in granular media. Chem. Engr. Sci. 1965. 20: 867-873

Yue X A, Hou J R, Qiu M J, et al. Evaluation of the character of profile control by polymer gelled particles. Petroleum Geology and Recovery Efficiency. 2006. 13(2): 81-84 (in Chinese)

Zhang H L and Liu H Q. Percolation performance of suspending profile control agent in reservoir formation. Journal of Hydrodynamics (Ser. A). 2007. 22(1): 113-118 (in Chinese)

Zuo K Z. Design of the polymer filtration coefficient meter and its application. Petroleum Instruments. 1998. 12(1): 22-26 (in Chinese)

(Edited by Sun Yanhua) 